\title{
La réception de la linguistique cognitive dans la linguistique du français
}

\author{
Dirk Geeraerts \\ Université Catholique de Louvain \\ dirk.geeraerts@arts.kuleuven.be
}

\section{L'essor de la linguistique cognitive}

La linguistique cognitive fait succès. En partant de la position relativement marginale qu'elle occupait initialement dans le paysage linguistique, elle est devenue un des courants majeurs de la linguistique contemporaine. Une recherche dans les Linguistics and Language Behavior Abstracts sur les termes cognitive linguistics et cognitive grammar, en comparaison avec generative grammar et generative linguistics, suggère que la popularité de la linguistique cognitive surpasse celle du générativisme : pour la période de cinq ans entre 2002 et 2007, la bibliographie inclut environ 300 références contenant les termes generative grammar ou generative linguistics, tandis qu'il y en a plus de 900 pour les termes cognitive linguistics et cognitive grammar. Pour la période de cinq ans entre 1988 et 1991, par contre, la bibliographie mentionne plus de 300 références pour la linguistique générative, et moins de 100 pour la linguistique cognitive.

La seconde moitié des années 90 en particulier marque le début de l'institutionalisation internationale de la linguistique cognitive. En témoignent les branches nationales de l'International Cognitive Lingusitics Association qui naissent dès le milieu des années 90: l'association espagnole en 1998, la finlandaise, la polonaise et la slaviste en 2001, la russe en 2004, l'allemande, la koréenne, la japonaise et l'association française Aflico en 2005, et enfin les associations britannique et chinoise en 2006.

En outre, les dix dernières années, une multitude d'introductions à la linguistique cognitive et d'ouvrages de référence a été publiée: Ungerer et Schmid en 1996, Dirven et Verspoor en 1998, Violi en 2001, Delbecque en 2002, Croft et Cruse en 2004, Evans et Green en 2006, la collection d'articles Basic Readings, rédigée par Geeraerts en 2006 et le volume accompagnant sous la rédaction de Kristiansen, Achard, Dirven et Ruiz de Mendoza, puis en 2007 un autre recueil d'articles, rédigé par Evans, Bergen et Zinken, et l'ample Handbook of Cognitive Linguistics, rédigé par Geeraerts et Cuyckens.

Etant donné cet essor de la linguistique cognitive, quelle est la contribution de l'étude linguistique du français dans ce développement? Partant de la polysémie de la notion 'linguistique française', faisons d'abord la distinction entre la linguistique du français et la linguistique en France. C'est la première qui nous intéresse ici : comment l'étude linguistique du français a-t-elle reçu et incorporé l'épanouissement international de la linguistique cognitive, ou bien, en renversant la perspective, quelle est la contribution de l'étude du français à la linguistique cognitive ? Nous nous proposons d'examiner la question en deux temps.

D'abord, une étude quantitative de l'apport de la linguistique française (dorénavant, nous entendrons par 'linguistique française' uniquement 'linguistique du français') à la linguistique cognitive nous permettra de comparer la réception de cette dernière en France, en Allemagne et en Espagne et de déterminer si oui ou non, la position de la linguistique française dans l'ensemble des études cognitivistes est comparable à celle qu'occupent les recherches cognitivistes dans deux traditions de recherche qui, culturellement et historiquement, sont comparables à la linguistique française - c'est-à-dire, des traditions de recherche en linguistique qui, par l'existence de théories et de méthodologies propres, ne se confondent pas entièrement avec la linguistique internationale de la seconde moitié du vingtième siècle.

Ensuite, une analyse qualitative des thématiques abordées par les études de linguistique française d'orientation cognitive nous permettra de déterminer s'il existe des préférences 'locales' dans la réception de la linguistique cognitive. Est-ce qu'il y a des thèmes qui ont reçu plus ou moins d'attention en 
linguistique française que dans la linguistique cognitive en général ? Est-ce que le champ d'application de la linguistique française d'orientation cognitive est semblable à celui de la linguistique cognitive internationale?

La base de données dont nous disposons pour mener notre recherche à bon terme consiste surtout en la Bibliography of Cognitive Linguistics (CogBib) rédigée par René Dirven et publiée comme annexe électronique de la revue Cognitive Linguistics. La version que nous employons est la dernière qui soit disponible en avril 2008, c'est-à-dire, la version qui recouvre une période jusqu'à la fin de l'année 2006 et qui contient 8987 références bibliographiques. Cette bibliographie n'est certainement pas exhaustive, mais elle nous semble assez détaillée et assez représentative pour entamer une analyse telle que nous l'envisageons. Comme matériaux supplémentaires, nous aurons recours aux Linguistics and Language Behavior Abstracts.

\section{L'analyse quantitative}

Constatons d'abord que la linguistique française est moins populaire en linguistique cognitive que les autres traditions linguistiques que nous nous proposons de comparer. Le Tableau 1 donne trois séries de chiffres tirées de la Bibliography of Cognitive Linguistics. La série MOTS CLES montre le nombre de références qui portent le mot clé French, German ou Spanish dans le champ Keywords de la base de données (tout en écartant des mots clés trompeurs comme Germanic). La série TITRE présente le nombre de références qui contiennent le mot français, deutsch ou español dans le titre de la publication (y compris des formes déclinées comme deutscher ou deutschen). La série MOTS CLES OU TITRE est la disjonction des deux séries préalables. (En raison du recouvrement entre les deux sous-ensembles MOTS CLES et TITRE, les chiffres pour la série MOTS CLES OU TITRE ne correspondent pas à la simple addition des chiffres précédents.)

Dans les trois séries, le classement est le même : la linguistique de l'allemand est la plus productive des trois, tandis que la linguistique française est la moins représentée.

\begin{tabular}{llll}
\hline & FRANÇAIS & ALLEMAND & ESPAGNOL \\
\hline MOTS CLES & 187 & 256 & 218 \\
TITRE & 15 & 39 & 21 \\
MOTS CLES OU TITRE & 191 & 258 & 225
\end{tabular}

Tableau 1

Notons que la position relativement faible de la linguistique française n'est probablement pas un effet de visibilité. La sous-représentation dans CogBib de la linguistique japonaise ou chinoise, par exemple, est certes due à la disponibilité et l'accessibilité réduite des publications japonaises et chinoises, mais un tel effet est difficile à concevoir pour les trois langues européennes qui entrent dans notre comparaison.

Par contre, on peut s'imaginer que la position du français relève d'un délai chronologique: peut-être que malgré la présence internationale, depuis les débuts du mouvement cognitiviste, de Gilles Fauconnier et Claude Vandeloise comme linguistes cognitifs d'origine francophone - la linguistique française ait assumé une perspective cognitive plus tard que les autres traditions.

Dans le Tableau 2, les chiffres de la série MOTS CLES sont répartis en six périodes chronologiques de cinq ans. La série $\mathrm{N}$ spécifie le nombre total de références pour chaque période dans la bibliographie. 


\begin{tabular}{lllllll}
\hline & $77-81$ & $82-86$ & $87-91$ & $92-96$ & $97-01$ & $02-06$ \\
\hline $\mathrm{N}$ & 247 & 591 & 1093 & 1647 & 2416 & 2557 \\
MOT CLE = FRENCH & 0 & 14 & 30 & 34 & 45 & 64 \\
MOT CLE = GERMAN & 7 & 13 & 33 & 48 & 80 & 72 \\
MOT CLE $=$ SPANISH & 0 & 2 & 13 & 36 & 74 & 91
\end{tabular}

Tableau 2

Il s'avère que la productivité de la linguistique espagnole est assez récente : ce n'est que depuis le milieu des années 90 que l'étude de l'espagnol occupe une position marquée dans l'ensemble des études cognitivistes. La linguistique française et la linguistique allemande, par contre, connaissent un développement plus ou moins comparable jusqu'au milieu des années 90 , mais à partir de ce moment, la linguistique française ne connaît pas la même croissance que la linguistique allemande ou espagnole.

\begin{tabular}{lllllll}
\hline & $77-81$ & $82-86$ & $87-91$ & $92-96$ & $97-01$ & $02-06$ \\
\hline $\mathrm{N}$ & 247 & 591 & 1093 & 1647 & 2416 & 2557 \\
MOT CLE $=$ FRENCH & $0 \%$ & $2,368 \%$ & $2,744 \%$ & $2,064 \%$ & $1,862 \%$ & $2,502 \%$ \\
MOT CLE $=$ GERMAN & $2,834 \%$ & $2,199 \%$ & $3,019 \%$ & $2,914 \%$ & $3,311 \%$ & $2,815 \%$ \\
MOT CLE $=$ SPANISH & $0 \%$ & $0,338 \%$ & $1,189 \%$ & $2,185 \%$ & $3,062 \%$ & $3,558 \%$
\end{tabular}

Tableau 3

Il semble donc bien y avoir un certain effet chronologique de stagnation, mais remarquons que c'est un effet assez faible, comme le démontre le Tableau 3, qui reprend le Tableau 2 en nombres relatifs (plus précisément, en pourcentages par rapport au nombre $\mathrm{N}$ de l'ensemble des références pour chaque période).

L'analyse chronologique que nous venons de faire doit être complétée d'une analyse géographique. Si nous constatons que l'étude du français est relativement moins populaire en linguistique cognitive que l'étude de l'allemand ou de l'espagnol, c'est peut-être parce que la linguistique cognitive est moins populaire en France, le centre naturel et traditionnel de la linguistique française. Quelle est donc la position de la linguistique cognitive dans la linguistique française française, c'est-à-dire, la linguistique du français en France?

Pour en savoir plus, regardons la bibliographie Linguistics and Language Behavior Abstracts, qui ne contient qu'un nombre restreint de publications en linguistique cognitive, mais qui nous permet d'identifier le pays de publication et l'affiliation des auteurs. Dans le Tableau 4, nous présentons deux séries de pourcentages. La série PROVENANCE montre la proportion des publications en linguistique cognitive qui ont été publiées en France, en français. (Le dénominateur de la proportion est identifié en fonction de l'apparition du terme cognitive linguistique dans n'importe quel champ de la bibliographie, en conjonction avec l'apparition de French, German ou Spanish dans le champ Keywords. Le numérateur est identifié à partir des champs Country et Language.) La série AFFILIATION indique la proportion des 
publications en linguistique cognitive qui ont été publiées par des auteurs avec une affiliation institutionnelle en France.

\begin{tabular}{lccc}
\hline & FRANÇAIS & ALLEMAND & ESPAGNOL \\
\hline PROVENANCE & $11.94 \%$ & $19.23 \%$ & $18.64 \%$ \\
AFFILIATION & $16.41 \%$ & $29.48 \%$ & $38.98 \%$
\end{tabular}

Tableau 4

La première série de chiffres montre que, plus que pour l'allemand ou l'espagnol, les études d'orientation cognitiviste sont publiées en dehors de la France ou dans des langues autres que le français. Cette observation est ambiguë, puisqu'elle signifie ou bien que les cognitivistes établis en France publient proportionnellement plus en anglais (ou d'autres langues) que les cognitivistes de l'Allemagne ou de l'Espagne, ou bien simplement qu'il y a proportionnellement moins de cognitivistes en France. La deuxième série de chiffres montre que cette dernière interprétation est la plus probable : les travaux d'orientation cognitiviste se trouvent plus souvent dans la périphérie géographique pour le français que pour l'allemand ou l'espagnol. On pourrait donc dire que la linguistique cognitive n'a pas encore pénétré autant dans le cour géographique de la linguistique française qu'elle ne l'a fait pour la linguistique allemande ou espagnole.

\section{L'analyse qualitative}

L'analyse quantitative semble suggérer que l'ancrage de la linguistique cognitive dans la linguistique française est un peu plus faible que dans la linguistique allemande et espagnole : la productivité est relativement moins élevée, il y a une certaine stagnation chronologique, et l'approche cognitiviste occupe une position moins forte dans le cœur géographique de la linguistique française. La question qui se pose est celle de savoir si cette situation spécifique se reflète dans le choix des sujets étudiés par les linguistes français d'orientation cognitiviste. Est-ce que la linguistique cognitive française se penche sur les mêmes thèmes que la linguistique cognitive en général, ou est-ce que la réception de la linguistique cognitive est thématiquement partielle?

Dans l'ensemble des références délimité par le critère MOTS CLES OU TITRE du Tableau 1, nous avons repéré dix sujets typiquement cognitivistes, en mesurant le nombre de références correspondant à chaque sujet. Voici les dix sujets, les abréviations que nous emploierons, et la liste des termes dont nous nous sommes servis pour la recherche des références:

- polysemy, vagueness, ambiguity

- radial network

- prototypicality, prototype theory

- spatial meaning

- metaphor

- image schema

- metonymy

- embodiment

- blending, mental spaces, conceptual integration

- construction grammar

$\begin{array}{ll}\text { PO } & \text { polysem*, vague*, ambigu* } \\ \text { RA } & \text { radial, network } \\ \text { PR } & \text { prototyp* } \\ \text { SP } & \text { spatial } \\ \text { MR } & \text { metaphor* } \\ \text { IM } & \text { image schema } \\ \text { MN } & \text { metonym* }^{*} \\ \text { EM } & \text { embod* } \\ \text { BL } & \text { blend*, mental space, conceptual integration } \\ \text { CN } & \text { construction }\end{array}$

Notons que ces sujets sont classés dans un ordre plus ou moins chronologique, qui correspond aux étapes du déploiement de la linguistique cognitive. Partant d'un intérêt pour l'architecture globale de la flexibilité sémantique (la polysémie, la prototypicalité, les réseaux radiaux - phénomènes dont le sens 
spatial sert souvent d'illustration), en passant par l'attention pour les mécanismes spécifiques de la polysémie, comme la métaphore et (chronologiquement plus tard) la métonymie, on arrive aux sujets dont la popularité est plus récente, comme la théorie des espaces mentaux et la grammaire constructionnelle.

\begin{tabular}{lllllllllll}
\hline & PO & RA & PR & SP & MR & IM & MN & EM & BL & CN \\
\hline FRANÇAIS & 14 & 13 & 16 & 41 & 44 & 4 & 14 & 0 & 8 & 38 \\
ALLEMAND & 15 & 12 & 21 & 41 & 54 & 9 & 13 & 2 & 5 & 48 \\
ESPAGNOL & 11 & 8 & 21 & 16 & 60 & 6 & 20 & 6 & 15 & 51
\end{tabular}

Tableau 5

Le Tableau 5 visualise les résultats de la recherche. Pour en arriver à une interprétation intuitivement claire des différences, le Graphique 1 fournit une représentation de type 'mosaïque'. La distribution des sujets selon l'axe horizontal montre la popularité des thèmes dans l'ensemble des matériaux pour les trois langues: la sémantique de la spatialité, la métaphore et la grammaire constructionnelle s'imposent comme sujets préférentiels. Ce qui est plus important, c'est la distribution des sujets selon la dimension verticale, qui montre des différences entre les trois langues. La linguistique espagnole prête relativement plus d'attention aux sujets récents, visualisés à droite de la représentation graphique, tandis que la linguistique française est surreprésentée à gauche, où se trouvent les thèmes cognitivistes 'classiques', comme la polysémie et la spatialité.

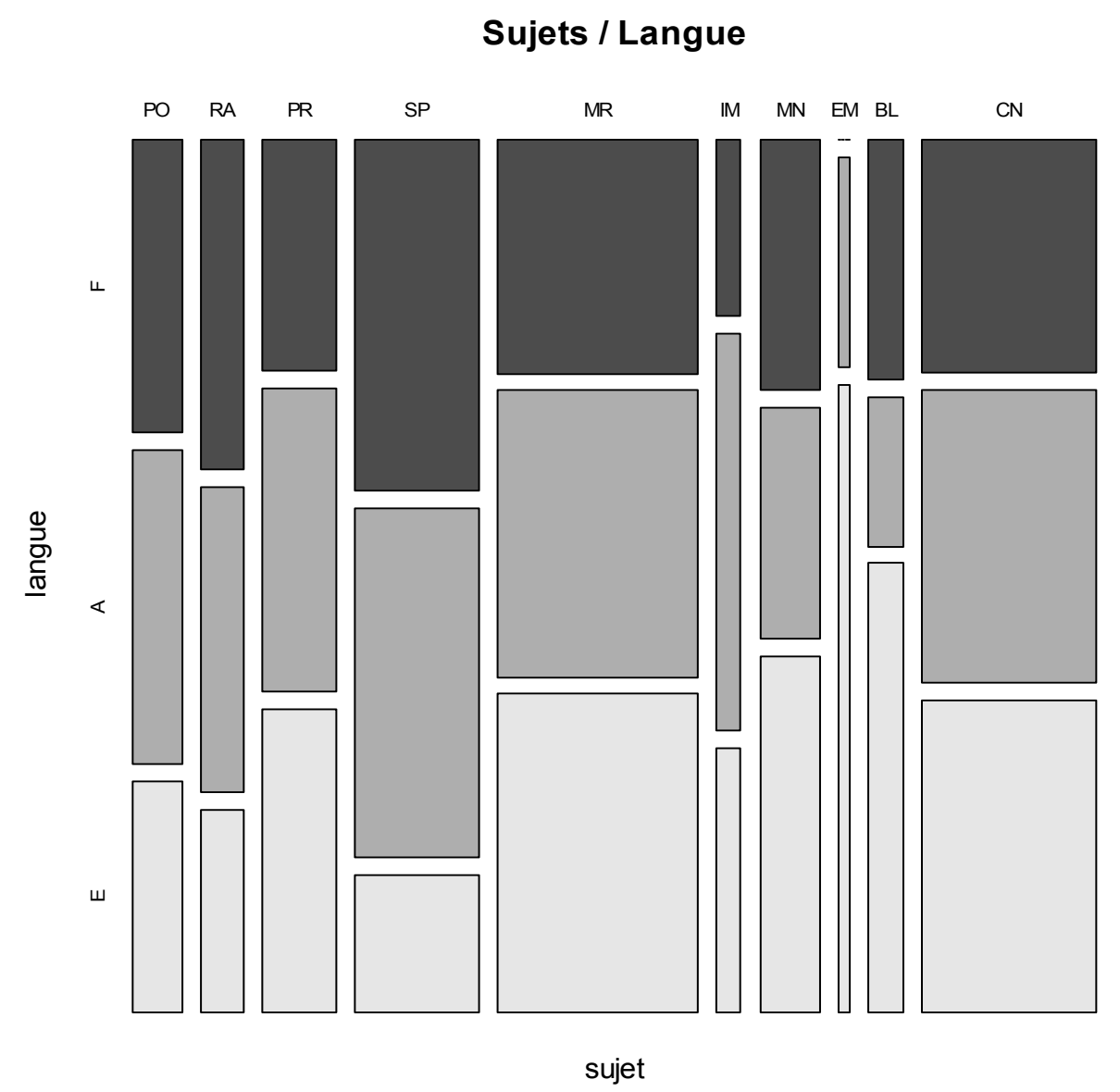




\section{Graphique 1}

Cette observation confirme l'analyse chronologique du Tableau 2. Le fait que la linguistique française n'a pas participé autant que la linguistique allemande ou espagnole à l'évolution explosive de la linguistique cognitive dès le milieu des années 90 , se reflète dans un certain degré de sous-représentation relative des sujets récents.

\section{Conclusions}

Nos analyses suggèrent que l'impact de la linguistique cognitive sur la linguistique du français n'est pas aussi profond que sur la linguistique de l'allemand ou de l'espagnol. En effet, dans la Bibliography of Cognitive Linguistics, le nombre d'articles qui étudient le français est relativement moins élevé que le nombre d'articles pour l'allemand ou l'espagnol. Il y a une certaine stagnation chronologique dans le développement de la linguistique cognitive française, ce qui se reflète dans les sujets étudiés par les linguistes français d'orientation cognitiviste : les sujets cognitivistes classiques sont surreprésentés en comparaison avec les sujets dont la popularité est plus récente. En plus, l'approche cognitiviste occupe une position plus faible en France, le cœur géographique de la linguistique française, par rapport à l'Allemagne ou l'Espagne.

Nous nous passons d'une spéculation sur les raisons de cette situation. Est-ce le résultat d'un manque d'accessibilité des publications cognitivistes ? Ou est-ce par contre le résultat d'une préférence délibérée pour d'autres théories linguistiques ? Constatons plutôt, de façon constructive et positive, qu'il y a encore du terrain cognitiviste à conquérir pour la linguistique du français.

\section{Références bibliographiques}

Croft, William \& D. Allan Cruse. 2004. Cognitive Linguistics. Cambridge: Cambridge University Press.

Delbecque, Nicole (éd.). 2002. Linguistique cognitive. Comprendre comment fonctionne le langage. Bruxelles: De Boeck Duculot.

Dirven, René \& Marjolein Verspoor (éd.). 1998. Cognitive Exploration of Language and Linguistics. Cognitive Linguistics in Practice 1. Amsterdam; Philadelphia, John Benjamins.

Evans, Vyvyan \& Melanie Green. 2006. Cognitive Linguistics: An Introduction. Mawhaw, N.J.; Edinburgh, U.K.: Lawrence Erlbaum; Edinburgh University Press.

Evans, Vyvyan, Benjamin Bergen \& Jörg Zinken (éd.). 2007. The Cognitive Linguistics Reader. London: Equinox.

Geeraerts, Dirk (éd.) 2006. Cognitive Linguistics: Basic Readings. Cognitive Linguistics Research. Berlin; New York, Mouton de Gruyter.

Geeraerts, Dirk \& Hubert Cuyckens (éd.). 2007. The Oxford Handbook of Cognitive Linguistics. New York: Oxford University Press.

Kristiansen, Gitte, Michel Achard, René Dirven \& Francisco Ruiz de Mendoza (éd.). 2006. Cognitive Linguistics: Current Applications, Future Orientations. Applications of Cognitive Linguistics 1. Berlin; New York, Mouton de Gruyter.

Ungerer, Friedrich \& Hans-Jörg Schmid. 1996. An Introduction to Cognitive Linguistics. London; New York: Longman.

Violi, Patrizia. 2001. Meaning and Experience. Bloomington, Ind.: Indiana University Press. 